\title{
AKARI NEAR-INFRARED SPECTROSCOPIC SURVEY FOR COMETARY VOLATILES
}

\author{
T. Ootsubo ${ }^{1}$, H. Kawakita ${ }^{2}$, H. Kobayashi ${ }^{2}$, F. Usui ${ }^{3}$, and AKARI SOSOS team \\ ${ }^{1}$ Astronomical Institute, Tohoku University, Aramaki, Aoba-ku, Sendai 980-8578, Japan \\ E-mail: ootsubo@astr.tohoku.ac.jp \\ ${ }^{2}$ Kyoto Sangyo University, Motoyama, Kamigamo, Kita-Ku, Kyoto 603-8555, Japan \\ ${ }^{3}$ Institute of Space and Astronautical Science, Japan Aerospace Exploration Agency, 3-1-1 Yoshinodai, Chuo-ku, \\ Sagamihara, Kanagawa 252-5210, Japan \\ (Received July 01, 2012; Accepted August 16, 2012)
}

\begin{abstract}
We performed a spectroscopic survey for cometary volatiles with the Infrared Camera onboard the Japanese infrared satellite AKARI. The observations were carried out in the near-infrared wavelength range in the period from 2008 June to 2010 January. In this paper, we summarize the observations and results of the AKARI survey for the mixing ratios of major volatiles in comets. We derived the 2.5-5 $\mu \mathrm{m}$ spectra of 18 comets including both Oort cloud comets and Jupiter-family comets. Prominent emission bands in the observed spectra are the fundamental vibrational bands of water $\left(\mathrm{H}_{2} \mathrm{O}\right)$ at $2.7 \mu \mathrm{m}$ and carbon dioxide $\left(\mathrm{CO}_{2}\right)$ at $4.3 \mu \mathrm{m}$. The fundamental vibrational band of carbon monoxide $(\mathrm{CO})$ at $4.7 \mu \mathrm{m}$ and the broad emission feature probably related to $\mathrm{C}-\mathrm{H}$ bearing molecules can also be recognized around the 3.4-3.5 $\mu \mathrm{m}$ region in some comets. We detect $\mathrm{CO}_{2}$ in 17 out of 18 comets, and derived gas production rate ratios of $\mathrm{CO}_{2}$ with respect to $\mathrm{H}_{2} \mathrm{O}$ in 17 comets. We detect a reliable $\mathrm{CO}$ emission band only in three of the comets. Our data set provides the largest homogeneous database of $\mathrm{CO}_{2} / \mathrm{H}_{2} \mathrm{O}$ ratios in comets obtained so far.
\end{abstract}

Key words: comets: general; infrared: planetary systems: protoplanetary disks

\section{INTRODUCTION}

Comets are considered to be the most pristine objects in the solar system. One of the main goals of cometary studies is the determination of the composition of volatile ice species contained in the nucleus. Chemical abundances from the cometary ices have been used to infer the conditions in the early solar nebula. One of the most important characteristics of cometary ice is the mixing ratio of major volatiles relative to water $\left(\mathrm{H}_{2} \mathrm{O}\right)$, especially for carbon dioxide $\left(\mathrm{CO}_{2}\right)$, carbon monoxide $(\mathrm{CO})$, and organics. Mixing ratios are thought to provide us with the precious information about the chemical evolution in the early solar nebula. Besides $\mathrm{H}_{2} \mathrm{O}, \mathrm{CO}_{2}$ and $\mathrm{CO}$ are the most abundant volatile species in cometary ices. However, because of the severe absorption of telluric $\mathrm{CO}_{2}$ in the atmosphere, we cannot access the cometary $\mathrm{CO}_{2}$ with ground-based observations. To date, the daughter species of $\mathrm{CO}_{2}$ have been used to determine the mixing ratio of $\mathrm{CO}_{2}$ (see Bockelée-Morvan et al., 2004). In addition, several direct measurements by spacecrafts (Combes et al., 1988; Feaga et al., 2007; A'Hearn et al., 2011) or infrared satellites (Crovisier et al., 1996, 1997, 1999a, 1999b; Colangeli et al., 1999) were available.

Recently, the Japanese infrared satellite AKARI (Murakami et al., 2007) has observed more than a dozen comets in the near-infrared wavelength region, and the mixing ratio of $\mathrm{CO}_{2}$ with respect to $\mathrm{H}_{2} \mathrm{O}$ for the comets are reported (Ootsubo et al., 2010; Ootsubo et al., 2012). In this paper, we summarize the observations and results of the AKARI survey for the mixing ratios of the major volatiles, in particular for $\mathrm{CO}_{2}$, in comets. As for the AKARI observations of the Solar System objects, the results of the asteroids (Usui et al., 
2012) and the zodiacal light (Pyo et al., 2012) are also reported in this volume.

\section{AKARI OBSERVATIONS OF COMETS}

The Japanese infrared satellite AKARI (Murakami et al., 2007) was launched on 2006 February 21, and its liquid helium (LHe) cryogen was exhausted on 2007 August 26 UT, 550 days after the launch. In the posthelium phase (called Phase 3), only near-infrared observations have been carried out after 2008 June (Onaka et al., 2010). Near-infrared observations of comets were performed with the Infrared Camera (IRC) onboard AKARI during this post-helium phase mainly as part of the SOSOS Mission Program observations, and several comets were observed as part of the AKARI director's time observations.

The IRC has a spectroscopic capability in both the grism (NG) mode and the prism (NP) mode with the AKARI IRC Astronomical Observation Template (AOT) IRCZ4 in Phase 3. The IRC NG and NP mode can cover the wavelength range from 2.5 to $5 \mu \mathrm{m}$ and from 1.8 to $5.5 \mu \mathrm{m}$, respectively (Onaka et al., 2007; Ohyama et al., 2007), where the vibrational bands of $\mathrm{H}_{2} \mathrm{O}, \mathrm{CO}_{2}$, and $\mathrm{CO}$ (at 2.7, 4.3, and $4.7 \mu \mathrm{m}$, respectively) are usually recognized as emission in the cometary spectra. The NG mode has a higher spectral resolution than the NP mode and it is suitable for observing the molecular emission bands. By contrast, the NP mode has a higher sensitivity than the NG mode.

Although several comets were observed with the $5^{\prime \prime}$ slit $(\mathrm{Ns})$, most of the comets were put on the $1^{\prime} \times 1^{\prime}$ aperture mask $(\mathrm{Np})$ with the IRC spectroscopy to observe the wide coma regions and minimize the contamination from nearby objects (Ootsubo et al., 2010; Ootsubo et al., 2012). We performed a very careful data reduction for the comets because the objects are moving targets and extended. We use second- or thirdorder polynomial functions to fit and subtract the continuum component from the spectra. Detailed information about the data reduction for the NG spectroscopic observations of comets is given in Ootsubo et al. (2012).

As a first step, we concentrate on the data taken in the IRC/NG mode with $\mathrm{Np}$ window $(2.5$ to $5 \mu \mathrm{m})$. From the 37 pointed NG observations (Table 1), we derived spectra for 18 comets and all the comet spectra are shown in Ootsubo et al. (2012). We also observed several comets in the NP mode, including other comets
TABLE 1.

Observed Comets with AKARI in NG Mode

\begin{tabular}{|c|c|c|c|}
\hline \multicolumn{4}{|c|}{ Jupiter-family Comets } \\
\hline OBJECT & UT Date & $\mathrm{r}_{h}[\mathrm{AU}]$ & $\Delta[\mathrm{AU}]$ \\
\hline 19P/Borrelly & 2008 Dec 30.08 & 2.19 & 1.95 \\
\hline $22 \mathrm{P} / \mathrm{Kopff}$ & 2009 Apr 22.57 & 1.61 & 1.26 \\
\hline $22 \mathrm{P} / \mathrm{Kopff}$ & 2009 Apr 22.63 & 1.61 & 1.26 \\
\hline $22 \mathrm{P} / \mathrm{Kopff}$ & 2009 Dec 11.18 & 2.42 & 2.22 \\
\hline $22 \mathrm{P} /$ Kopff & 2009 Dec 11.45 & 2.43 & 2.22 \\
\hline $22 \mathrm{P} / \mathrm{Kopff}$ & 2009 Dec 11.52 & 2.43 & 2.22 \\
\hline 29P/Schwassmann-Wachmann & 2009 Nov 18.49 & 6.18 & 6.09 \\
\hline 29P/Schwassmann-Wachmann & 2009 Nov 18.56 & 6.18 & 6.09 \\
\hline $64 \mathrm{P} /$ Swift-Gehrels & 2009 Nov 23.09 & 2.27 & 2.05 \\
\hline $64 \mathrm{P} /$ Swift-Gehrels & 2009 Nov 23.16 & 2.27 & 2.05 \\
\hline 67P/Churyumov-Gerasimenko & 2008 Nov 02.38 & 1.84 & 1.56 \\
\hline 81P/Wild & 2009 Dec 14.10 & 1.74 & 1.44 \\
\hline 81P/Wild & 2009 Dec 14.16 & 1.74 & 1.44 \\
\hline $81 \mathrm{P} / \mathrm{Wild}$ & 2009 Dec 14.50 & 1.74 & 1.43 \\
\hline 88P/Howell & 2009 Jul 03.06 & 1.74 & 1.41 \\
\hline 88P/Howell & 2009 Jul 03.13 & 1.73 & 1.41 \\
\hline 116P/Wild & 2009 May 15.60 & 2.22 & 1.98 \\
\hline 116P/Wild & 2009 May 16.49 & 2.22 & 1.99 \\
\hline 118P/Shoemaker-Levy & 2009 Sep 08.71 & 2.18 & 1.93 \\
\hline 118P/Shoemaker-Levy & 2009 Sep 08.78 & 2.18 & 1.93 \\
\hline 144P/Kushida & 2009 Apr 18.48 & 1.70 & 1.37 \\
\hline 144P/Kushida & 2009 Apr 18.55 & 1.70 & 1.37 \\
\hline 157P/Tritton & 2009 Dec 30.13 & 1.48 & 1.11 \\
\hline 157P/Tritton & 2009 Dec 30.27 & 1.48 & 1.11 \\
\hline \multicolumn{4}{|c|}{ Oort Cloud Comets } \\
\hline OBJECT & UT Date & $\mathrm{r}_{h}[\mathrm{AU}]$ & $\Delta[\mathrm{AU}]$ \\
\hline C/2006 OF2 (Broughton) & 2008 Sep 16.72 & 2.43 & 2.21 \\
\hline C/2006 OF2 (Broughton) & 2009 Mar 28.07 & 3.20 & 3.04 \\
\hline C/2006 Q1 (McNaught) & 2008 Jun 03.59 & 2.78 & 2.59 \\
\hline C/2006 Q1 (McNaught) & 2009 Feb 23.76 & 3.64 & 3.50 \\
\hline C/2006 W3 (Christensen) & 2008 Dec 21.07 & 3.66 & 3.52 \\
\hline C/2006 W3 (Christensen) & 2009 Jun 16.75 & 3.13 & 2.96 \\
\hline C/2007 G1 (LINEAR) & 2008 Aug 20.23 & 2.80 & 2.62 \\
\hline C/2007 N3 (Lulin) & 2009 Feb 05.57 & 1.28 & 0.80 \\
\hline C/2007 N3 (Lulin) & 2009 Mar 30.67 & 1.70 & 1.36 \\
\hline C/2007 Q3 (Siding Spring) & 2009 Mar 03.27 & 3.29 & 3.14 \\
\hline C/2008 Q3 (Garradd) & $2009 \mathrm{Jul} 05.60$ & 1.81 & 1.48 \\
\hline C/2008 Q3 (Garradd) & 2009 Jul 06.49 & 1.81 & 1.50 \\
\hline C/2008 Q3 (Garradd) & 2010 Jan 03.14 & 2.96 & 2.78 \\
\hline
\end{tabular}

that are not listed in Table 1, not only with the NG mode. The results of the comets observed in the NP mode will be discussed elsewhere in the near future.

\section{MIXING RATIOS}

We detect $\mathrm{H}_{2} \mathrm{O}$ in all comets, and $\mathrm{CO}_{2}$ in 17 out of 18 comets except for the comet 29P/SchwassmannWachmann 1 . In case of the comet $29 \mathrm{P}$, the spectra were obtained when the object was at around 6 AU from the Sun, and the $\mathrm{CO}_{2}$ emission was not detected on a $3 \sigma$ level. This is probably explained by the insufficient evaporation of $\mathrm{CO}_{2}$ from the ice $\left(\mathrm{T}_{\text {sub }} \sim 70 \mathrm{~K}\right)$, which is in contrast to $\mathrm{CO}\left(\mathrm{T}_{\text {sub }} \sim 25 \mathrm{~K}\right)$. We only detect a reliable $\mathrm{CO}$ emission band in three of the comets, including the comet 29P. The small number of secure $\mathrm{CO}$ detection in the comets is caused by the small fluo- 
rescence efficiency of the $\mathrm{CO}$ fundamental band at 4.67 $\mu \mathrm{m}$. We need higher wavelength resolution and sensitivity than AKARI/IRC for the secure detection of $\mathrm{CO}$ at $4.7 \mu \mathrm{m}$.

We use the single-generation Haser model (Haser, 1957) to estimate the gas production rate from the observed molecular band flux for $\mathrm{H}_{2} \mathrm{O}, \mathrm{CO}_{2}$, and $\mathrm{CO}$. The number density of the molecule " $\mathrm{X}$ " $\left(n_{\mathrm{X}}\right)$ is represented by

$$
n_{\mathrm{X}}(\rho)=\frac{Q(\mathrm{X})}{4 \pi v_{\exp } \rho^{2}} \exp \left(-\frac{\rho}{v_{\exp } \tau_{\mathrm{X}}}\right),
$$

where $Q(\mathrm{X})$ is the gas production rate of the molecule $\mathrm{X}$ (molecules $\left.\mathrm{s}^{-1}\right), v_{\exp }$ is the expansion velocity of the gas, $\rho$ is the nucleocentric distance, and $\tau_{\mathrm{x}}$ is the photodissociation lifetime of the molecule X (see BockeléeMorvan et al., 2004 and Ootsubo et al., 2012 for more details).

Based on the gas production rate of $\mathrm{H}_{2} \mathrm{O}$ and $\mathrm{CO}_{2}$ obtained here, we derived the gas production rate ratios (the mixing ratios) of $\mathrm{CO}_{2}$ with respect to $\mathrm{H}_{2} \mathrm{O}$. Figure 1 illustrates the mixing ratios of $\mathrm{CO}_{2}$ with respect to $\mathrm{H}_{2} \mathrm{O}$ in the 13 comets observed within 2.5 AU from the Sun, where $\mathrm{H}_{2} \mathrm{O}$ effectively sublimates from the nucleus of the comet. The difference between the Oort cloud comets (OCs) and the Jupiter-family comets (JFCs) is emphasized in different colors. The mixing ratio of $\mathrm{CO}_{2}$, with respect to $\mathrm{H}_{2} \mathrm{O}$, spans from several to $\sim 30 \%$ in our sample of both the JFCs and the OCs.

In Figure 1, we plot the results of the previous studies for the comet $1 \mathrm{P} /$ Halley, 9P/Tempel, and 103P/Hartley, together with our result. The results of these measurements fall within the range of our measurements. The present data set of the large samples confirms the range of the ratios obtained by previous measurements (Bockelée-Morvan et al., 2004).

\section{COMPARISON WITH PROTOSTELLAR SAM- PLES}

The comparison in the mixing ratios of ices between the comets and protostellar samples is important because it is the key to answer the essential questions in comet science. It is still an open question whether or not the interstellar ices were chemically processed in the solar nebula before they were incorporated into the cometary nuclei. As for the $\mathrm{CO}_{2}$, the AKARI survey gives us the first opportunity to perform such comparison.

The results for 13 comets observed within $2.5 \mathrm{AU}$

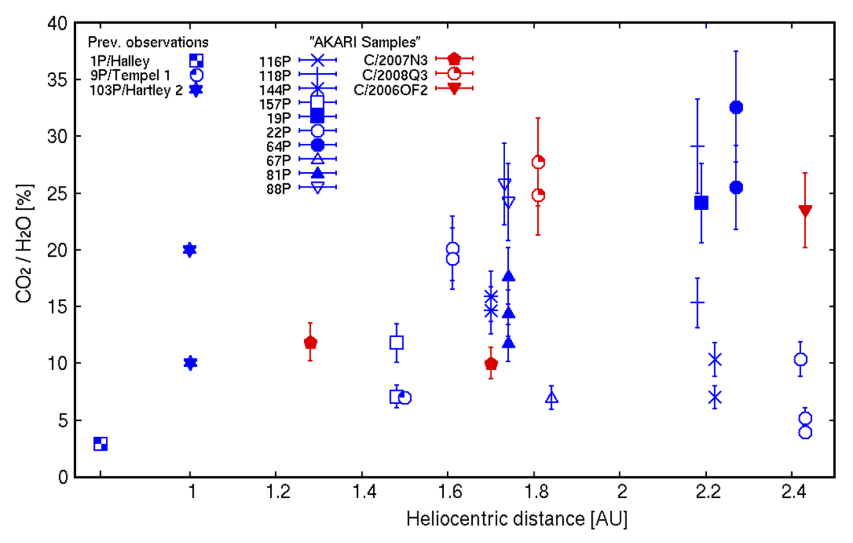

Fig. 1. The mixing ratios of $\mathrm{CO}_{2}$ with respect to $\mathrm{H}_{2} \mathrm{O}$ in the 13 comets observed with AKARI/IRC within 2.5 AU from the Sun. The difference between Oort cloud comets (red) and Jupiter-family comets (blue) are emphasized with different colors. The results obtained with the previous studies before AKARI (1P/Halley, 9P/Tempel, and $103 \mathrm{P} /$ Hartley) are also depicted in the figure.

from the Sun show that the range of the $\mathrm{CO}_{2} / \mathrm{H}_{2} \mathrm{O}$ ratio in the comets represented by the upper and lower quartile values is $11 \%-24 \%$ (the median value is $17 \%$ ). In the protostellar samples, the $\mathrm{CO}_{2} / \mathrm{H}_{2} \mathrm{O}$ ratios are $22 \%-35 \%$ (the median value is $29 \%$ ) and $12 \%-22 \%$ (the median value is 13\%) as the upper and lower quartile values in the low-mass and high-mass protostar envelopes, respectively (Öberg et al., 2011). The $\mathrm{CO}_{2}$ in the cometary ice is more depleted with respect to water and more diverse with respect to the median abundance than the low-mass protostellar ices. The $\mathrm{CO}_{2} / \mathrm{H}_{2} \mathrm{O}$ ratios in comets seem to be comparable with those in the high-mass protostellar ices rather than with the low-mass protostellar envelope ices. The processes that take place in the high-mass protostar envelopes might also have occurred in the solar nebula disk, such as heating and UV processing by the Sun (Ootsubo et al., 2012, for more detailed discussions).

\section{CONCLUDING REMARKS}

Both Oort cloud comets and Jupiter-family comets were observed spectroscopically with the Infrared Camera onboard the Japanese infrared satellite AKARI in the period from 2008 June to 2010 January. With the grism mode in the wavelength range from 2.5 to $5 \mu \mathrm{m}$, we derived the spectra of 18 comets from 37 pointed observations. Our data set provides the largest homogeneous database of $\mathrm{CO}_{2} / \mathrm{H}_{2} \mathrm{O}$ production rate ratios 
in comets obtained so far. Prominent emission bands of the fundamental vibrational bands of $\mathrm{H}_{2} \mathrm{O}$ at $2.7 \mu \mathrm{m}$ and $\mathrm{CO}_{2}$ at $4.3 \mu \mathrm{m}$ can be seen in all comet spectra except for comet 29P/Schwassmann-Wachmann 1 .

The gas production rate ratios of $\mathrm{CO}_{2}$ with respect to $\mathrm{H}_{2} \mathrm{O}$ have been derived for 17 comets. The $\mathrm{CO}_{2} / \mathrm{H}_{2} \mathrm{O}$ ratio in cometary ice spans from several to $\sim 30 \%$ among the comets observed within $2.5 \mathrm{AU}$ from the Sun. The range of $\mathrm{CO}_{2} / \mathrm{H}_{2} \mathrm{O}$ ratios is represented by the upper and lower quartile values of $11 \%-24 \%$ (the median value is $17 \%$ ). These values are comparable to the ones from high-mass protostellar ices (12\%-22\%). $\mathrm{CO}_{2}$ in cometary ice is more depleted with respect to water and more diverse than in low-mass protostellar ices. The ices incorporated into comets should have been altered in the early solar nebula, such as the sublimation of $\mathrm{CO}_{2}$ ice by heating and UV processing by the Sun, assuming that the cometary ice composition has not been altered significantly after the formation of cometary nuclei.

$\mathrm{CO}$ was detected only in very few cases. We need a larger comet sample with good $\mathrm{CO}_{2}$ and $\mathrm{CO}$ detctions for further discussions.

\section{ACKNOWLEDGEMENTS}

This work is based on observations with AKARI, a JAXA project with the participation of ESA. We thank all members of the AKARI project for their continuous help and support. This work is supported in part by Grant-in-Aid for Young Scientists (B) No. 21740153 (T.O.) from the Ministry of Education, Culture, Sports, Science and Technology of Japan, and the Brain Circulation Program (R2301) from the Japan Society for the Promotion of Science.

\section{REFERENCES}

A'Hearn, M. F., et al., 2011, EPOXI at Comet Hartley 2, Science, 332, 1396

Bockelée-Morvan, D., Crovisier, J., Mumma, M. J., \& Weaver, H. A., 2004, The Composition of Cometary Volatiles, in Comets II, Vol. 745, Ed. M. C. Festou, H. U. Keller, \& H. A. Weaver (Tucson, AZ: Univ. Arizona Press), 391

Colangeli, L., et al., 1999, Infrared Spectral Observations of Comet 103P/Hartley 2 by ISOPHOT, A\&A, 343, L87

Combes, M., Crovisier, J., Encrenaz, T., Moroz, V. I., \& Bibring, J. -P., 1988, The 2.5-12 Micron Spec- trum of Comet Halley from the IKS-VEGA Experiment, Icarus, 76, 404

Crovisier, J., et al., 1996, The Infrared Spectrum of Comet C/1995 O1 (Hale-Bopp) at 4.6 AU from the Sun, A\&A, 315, L385

Crovisier, J., et al., 1997, The Spectrum of Comet Hale-Bopp (C/1995 01) Observed with the Infrared Space Observatory at 2.9 AU from the Sun, Science, 275, 1904

Crovisier, J., et al., 1999a, The Spectrum of Comet Hale-Bopp as Seen by ISO, in The Universe as Seen by $I S O$, Ed. P. COX \& M. F. Kessler (ESASP 427; Noordwijk: ESA), 137

Crovisier, J., et al., 1999b, ISO Spectroscopic Observations of Short-Period Comets, in The Universe as Seen by ISO, Ed. P. COX \& M. F. Kessler (ESA-SP 427; Noordwijk: ESA), 161

Feaga, L. M., et al., 2007, Asymmetries in the Distribution of $\mathrm{H}_{2} \mathrm{O}$ and $\mathrm{CO}_{2}$ in the Inner Coma of Comet $9 \mathrm{P} /$ Tempel 1 as Observed by Deep Impact, Icarus, 190, 345

Haser, L., 1957, Distribution D'intensite Dans la Tete d'une Comete, Bulletin de la Societe Royale des Sciences de Liege, 43, 740

Murakami, H., et al., 2007, The Infrared Astronomical Mission AKARI, PASJ, 59, S369

Öberg, K. I., et al., 2011, The Spitzer Ice Legacy: Ice Evolution from Cores to Protostars, ApJ, 740, 109

Ohyama, Y., et al., 2007, Near-Infrared and MidInfrared Spectroscopy with the Infrared Camera (IRC) for AKARI, PASJ, 59, S411

Onaka, T., et al., 2007, The Infrared Camera (IRC) for AKARI - Design and Imaging Performance, PASJ, 59, S401

Onaka, T., et al., 2010, AKARI Warm Mission, SPIE, $7731,77310 \mathrm{M}$

Ootsubo, T., et al., 2010, Detection of Parent $\mathrm{H}_{2} \mathrm{O}$ and $\mathrm{CO}_{2}$ Molecules in the 2.5-5 $\mu \mathrm{m}$ Spectrum of Comet C/2007 N3 (Lulin) Observed with AKARI, ApJ, 717, L66

Ootsubo, T., et al., 2012, AKARI Near-infrared Spectroscopic Survey for $\mathrm{CO}_{2}$ in 18 Comets, ApJ, 752, 15

Pyo, J., et al., 2012, Zodiacal Light in the Infrared from the Space Missions, in this volume

Usui, J., et al., 2012, A Panoramic View of the Asteroids in the Inner Solar System with AKARI, in this volume 